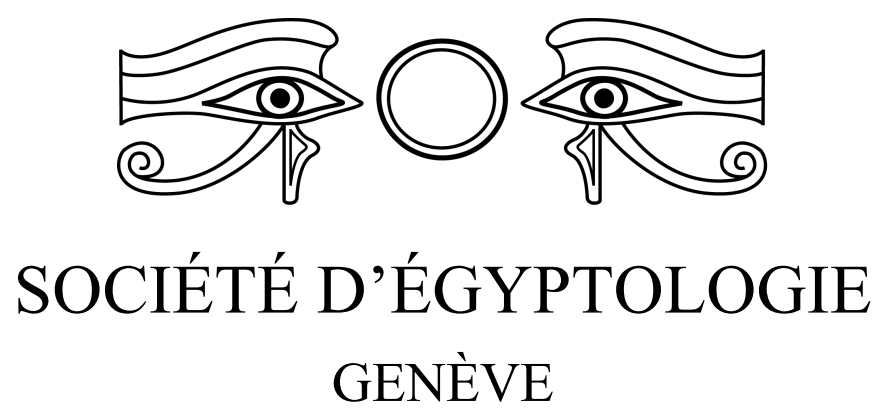

BULLETIN N ${ }^{\circ} 32$

\title{
La désignation du maître de Siwa à la Basse Époque et sa signification
}

\section{Olivier PERDU}

Collège de France

Institut des Civilisations

operdu@yahoo.fr

\section{Résumé}

Les diverses opinions exprimées sur la façon dont les chefs de Siwa contemporains des $\mathrm{XXVI}^{\mathrm{e}}$ et $\mathrm{XXX}^{\mathrm{e}}$ dynasties sont désignés ont conduit à réexaminer cette question et à préciser la portée de la solution retenue.

Mots-clés : Siwa; Basse Époque ; lexicographie

\author{
Rue du Cardinal Lemoine 52 \\ F-75231 PARIS Cedex 05
}

\begin{abstract}
The various opinions expressed on the manner in which the contemporary Siwa chiefs of the 26th and 30th dynasties are designated have led to a re-examination of this question and to a clarification of the scope of the solution adopted.
\end{abstract}

Keywords: Siwa; Late Period; lexicography

\section{Comment citer/How to cite}

Olivier PERDU, « La désignation du maître de Siwa à la Basse Époque et sa signification », BSÉG 32 (2021), pp. 79-90. 


\title{
La désignation du maître de Siwa à la Basse Époque et sa signification
}

\author{
Olivier Perdu
}

Les diverses opinions exprimées sur la façon dont les chefs de Siwa contemporains des $\mathrm{XXVI}^{\mathrm{e}}$ et $\mathrm{XXX}^{\mathrm{e}}$ dynasties sont désignés ont conduit à réexaminer cette question et à préciser la portée de la solution retenue.

Les sources pharaoniques de Basse Époque conservent le souvenir de quatre personnages pourvus d'un titre les désignant comme des maîtres de Siwa: Reroutek $^{1}$, ses fils Sethirdis (fig. 1) et Imenemipet ${ }^{2}$, et Ounamon ${ }^{3}$ (fig. 2). Les trois premiers, connus par le saint des saints du temple d'Aghourmi ${ }^{4}$ et une petite base d'ex-voto ${ }^{5}$, sont contemporains de la fin de la période saïte alors que le quatrième, attesté dans les sanctuaires d'Oum-Ebeyda ${ }^{6}$ et El-Bahreïn ${ }^{7}$, a été en fonction à la $\mathrm{XXX}^{\mathrm{e}}$ dynastie. Le titre qu'ils ont en commun est composé du substantif $w r$, 《chef»-écrit ou ${ }^{8}$ en étant éventuellement suivi du qualificatif 3 , « grand »- et d'un génitif se présentant sous la forme d'un mot au pluriel formé

\footnotetext{
${ }^{1}$ Suivant la lecture proposée par Fr. COLIn, dans W. ClARYSSE - A. SCHOORS - H. Willems (éds), Egyptian Religion, the Last Thousand Years: Studies Dedicated to the Memory of Jan Quaegebeur, I (OLA 84), Louvain 1998, p. 343, n. IV.

2 À supposer que son père soit à identifier à celui de Sethirdis qui a le même nom, comme la

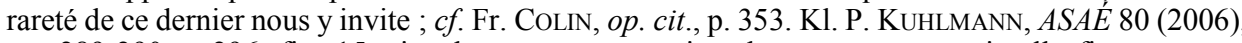
pp. 289-290 et 296, fig. 15, signale une autre mention du personnage, mais elle figure sur un fragment de relief où son titre n'apparaît pas distinctement.

${ }^{3}$ Son père est présenté comme son homologue, mais lui-même n'est jamais mentionné avec le titre en question.

${ }^{4}$ Voir A. FAKHRY, Siwa Oasis: Its History and Antiquities, Le Caire 1944, pp. 91-95.

${ }^{5}$ Voir Fr. COLIN, op. cit., p. 341.

${ }^{6}$ Voir A. FAKHRY, op. cit., pp. 102, 111 et 114.

${ }^{7}$ Voir P. Gallo, BSFÉ 166 (juin 2006), pp. 17, fig. 5, 25, fig. 12, et 26, fig. 13 et 14.

${ }^{8}$ Graphie uniquement attestée sur la base d'ex-voto.
} 

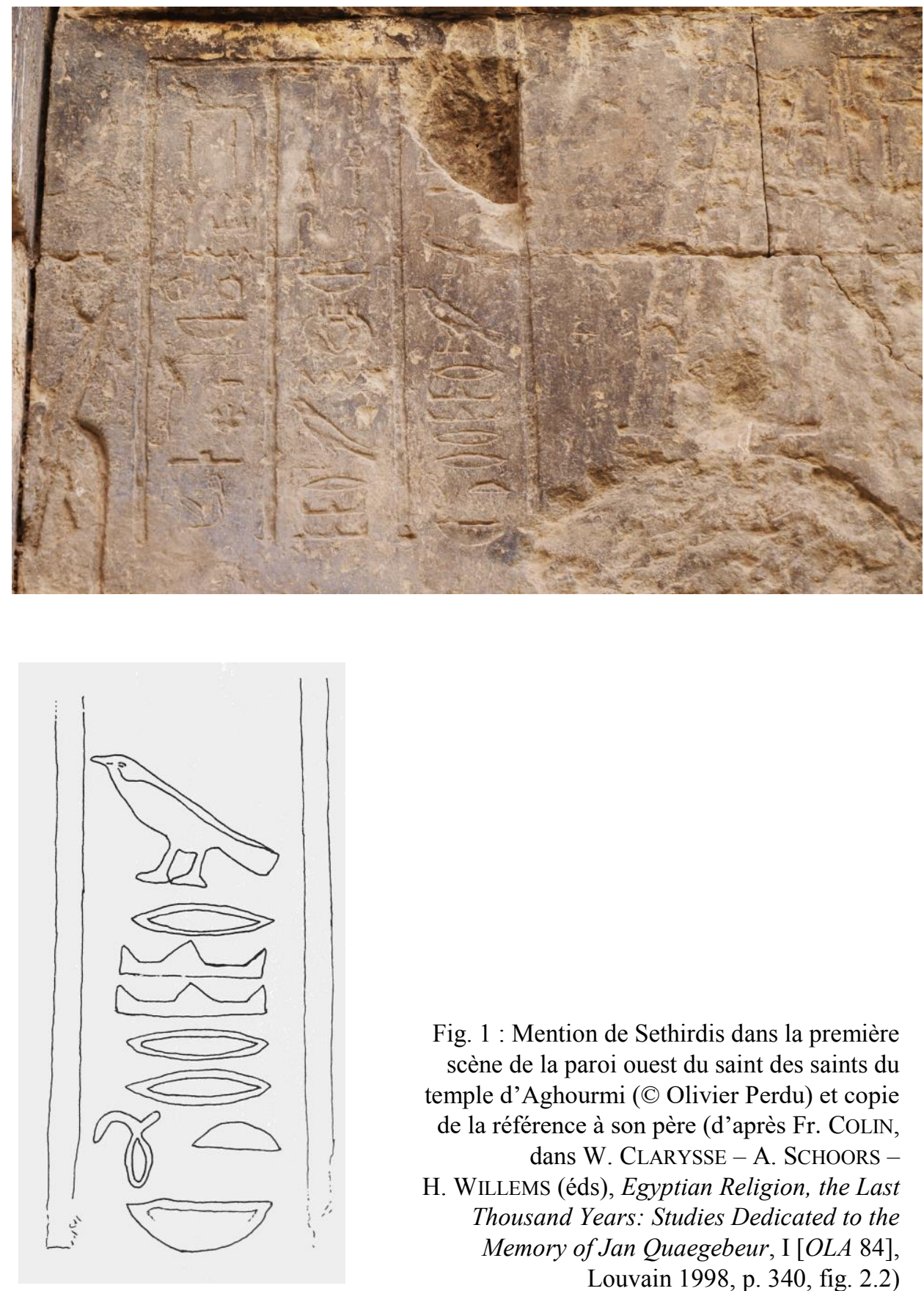

Fig. 1 : Mention de Sethirdis dans la première scène de la paroi ouest du saint des saints $d u$ temple d'Aghourmi ( $\subset$ Olivier Perdu) et copie de la référence à son père (d'après Fr. CoLIN, dans W. CLARYSSE - A. SCHOORS H. WiLLEMS (éds), Egyptian Religion, the Last Thousand Years: Studies Dedicated to the Memory of Jan Quaegebeur, I [OLA 84], Louvain 1998, p. 340, fig. 2.2) 
à partir du logogramme se lisant $h 3 s t^{9}$. Le problème qu'il pose tient à ce dernier élément dans la mesure où ses graphies ont donné lieu à plusieurs interprétations.

Bien qu'elles se rejoignent en dédoublant le signe du terrain vallonné, les graphies saïtes témoignent d'une certaine variété en se limitant à cet ensemble (a) ou en le faisant suivre de deux (b) ou trois traits (c):

a) $\simeq$ (5 ex. : A. FAKHRY, op. cit., pp. 92, 93, 94 et 95 ; Fr. COLIN, op. cit., p. 341)

b) $\frac{\pi}{11}(1$ ex. : Fr. Colin, loc. cit.)

c) $\frac{\pi}{111}(4$ ex. : A. FAKHRY, op. cit., pp. 91, 93 et 94)

Celles du IV $\mathrm{e}^{\mathrm{e}}$ siècle avant notre ère se signalent en revanche par leur uniformité en se réduisant au signe du terrain vallonné accompagné par les trois traits du pluriel (d) :

d) $\simeq$ (10 ex. : A. FAKHRY, op. cit., pp. 102, fig. 12, col. 8, 13, 21, 22, 31 et $37,111^{10}$ et 114 ; P. GALLO, op. cit., pp. 25 , fig. 12 , et 26 , fig. 14)

Ce que Ahmed Fakhry a traduit en 1944 par « Desert dwellers » ${ }^{11}$ ou « Foreign Lands $»^{12}$, Klaus P. Kuhlmann a proposé en 1988 de le lire ḩswt, « Fremdländer $»^{13}$, mais sans expliquer les exemples où le mot revêt l'aspect d'un duel ( $a$ et b). Cela pourrait éventuellement se concevoir dans la perspective d'un duel devenu une façon de noter le pluriel une fois oublié comme catégorie grammaticale ${ }^{14}$. On dispose notamment depuis le Nouvel Empire d'exemples où le pluriel d'un substantif écrit au moyen d'un seul signe - logogramme ou

\footnotetext{
${ }^{9}$ Noter cependant que J. OsING, $L \ddot{A} \mathrm{~V}^{7}, 1984$, col. 966, voit dans les exemples saïtes et ceux plus récents deux titres différents se lisant respectivement $w r$ h3stjw, "Fürst der Fremdländbewohner », et $w r$ [3] h3swt, " (großer) Fürst der Fremdländer ». Une distinction est également établie par J. MoJE, Herrschaftsräume und Herrschaftswissen ägyptischer Lokalregenten: Soziokulturelle Interaktionen zur Machtkonsolidierung vom 8. bis zum 4. Jahrhundert v. Chr., Berlin-Boston 2014, pp. 27, 211-212, 421-423 et 425-427, qui lit les premiers wr h3s.tj, et les autres $w r$ [3] h3s.wt.

${ }^{10}$ Photo et facsimilé de cet exemple dans P. Gallo, op. cit., pp. 28-29, fig. 15 et 16.

${ }^{11}$ Voir A. FAKHRY, op. cit., pp. 91-94 ; ID., BÄBA 12 (1971), p. 27.

12 Voir IBID., pp. 95 et 111

${ }^{13}$ Cf. K. P. Kuhlmann, Das Ammoneion: Archäologie, Geschichte und Kultpraxis des Orakels von Siwa (AVDAIK 75), Mayence 1988, pp. 103-105. Proposition reprise par G. ViTTMANN, Ägypten und die Fremden im ersten vorchristlichen Jahrtausend, Mayence 2003, pp. 17 et 19 et J. MoJE, op. cit., pp. 27-28, 212 et 425-427 (pour les exemples de la XXX ${ }^{\mathrm{e}}$ dynastie).

${ }^{14}$ Cf. C. Peust, Das Napatanische. Ein ägyptischer Dialekt aus den Nubien des späten ersten vorchristlichen Jahrtausends: Texte, Glossar, Grammatik, Göttingen 1999, p. 234.
} 

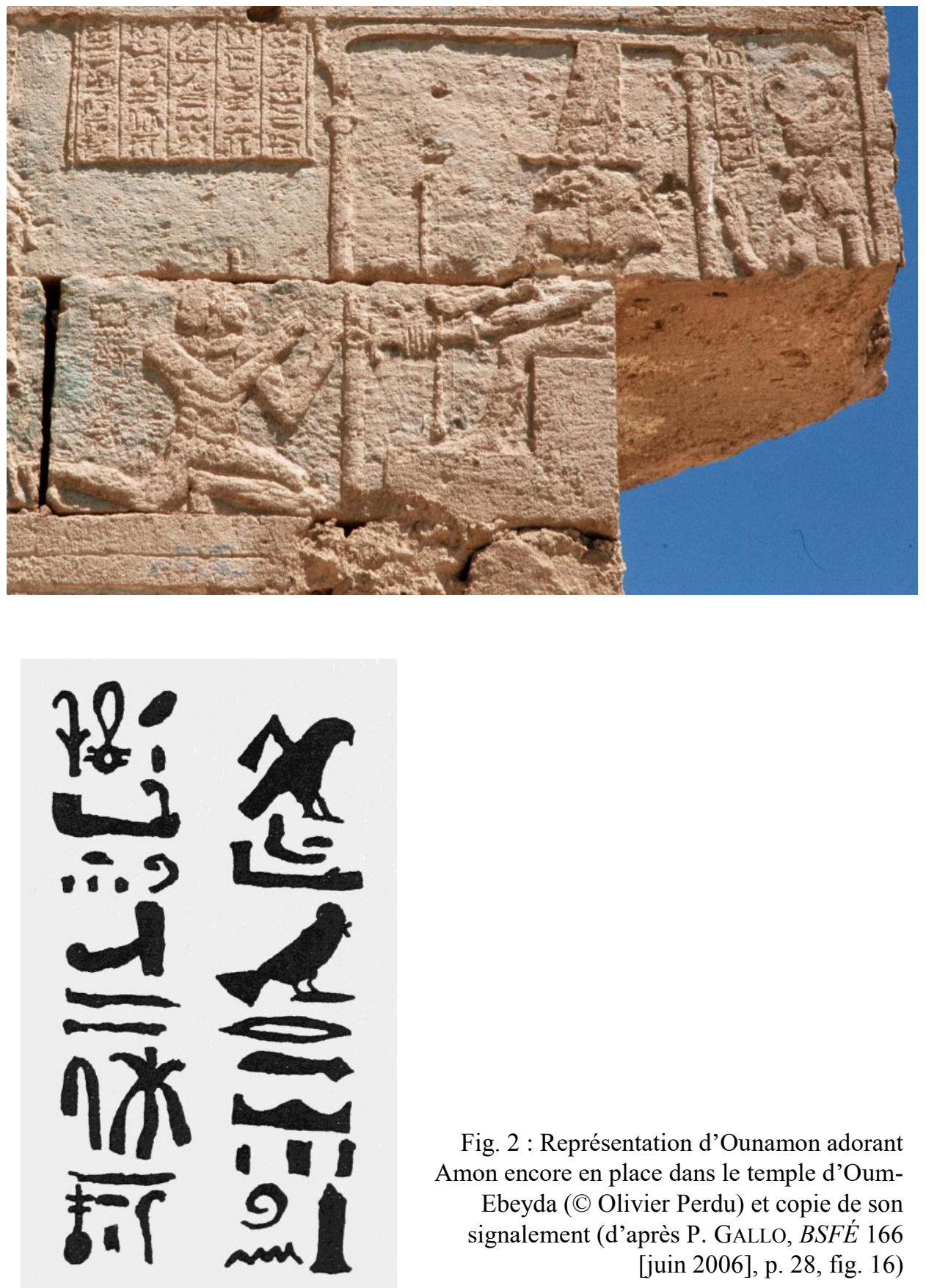

Fig. 2 : Représentation d'Ounamon adorant Amon encore en place dans le temple d'OumEbeyda (C) Olivier Perdu) et copie de son signalement (d'après P. GALLO, BSFÉ 166 [juin 2006], p. 28, fig. 16) 
radicogramme - est noté en le répétant deux fois au lieu de trois ${ }^{15}$. Cette explication semble néanmoins difficile à retenir dans le cas présent car les multiples graphies de h3swt rencontrées aux époques tardives n'ont jamais l'apparence d'un duel. Toutes se présentent comme d'authentiques pluriels dont les formes les plus courantes se limitent à trois : : 111,111 et 16 .

Dix ans plus tard, Frédéric Colin a envisagé une autre possibilité en lisant l'élément litigieux h3sty, les « Deux Confins », ce qui l'amène à rapprocher le titre où il apparait de celui désignant le pharaon comme le « maître des Deux Terres » $(n b t 3 w y)^{17}$. Sa proposition s'appuie sur le redoublement du logogramme de h3st, mais cette particularité, contrairement à ce qu'il affirme, se limite en fait aux exemples de la XXVI ${ }^{\mathrm{e}}$ dynastie (a-c). Les plus récents associent au contraire la désinence du pluriel au signe du terrain vallonné, les exemples relevés récemment à El-Bahreïn n'étant pas différents de ceux d'Oum-Ebeyda (d). On admettra par ailleurs que si le titre devait être un pastiche de " maître des Deux Terres », il serait surprenant de le rencontrer aussi souvent avec des graphies où le duel de h3st n'est pas noté - contrairement à celui de $t 3$ dans $n b t 3 w y$ - en répétant simplement le logogramme, notamment quand elles se rapportent à un personnage comme Ounamon, qui cherche précisément à s'assimiler à un pharaon dans ses présentations $^{18}$.

Tout en maintenant cette interprétation, son auteur a pensé à une autre éventualité en 2011 en suggérant cette fois de voir dans le redoublement du signe du terrain vallonné un « duel apparent » destiné à noter la finale en -ty du nisbé formé sur $h 3 s t^{19}$. Même si son hypothèse ne vaut que pour les exemples concernés par le redoublement, soit ceux de la XXVI ${ }^{\mathrm{e}}$ dynastie (a-c), elle est parfaitement recevable. La graphie adoptée par les exemples plus récents, ceux de la $\mathrm{XXX}^{\mathrm{e}}$ dynastie, peut en effet se concilier avec la perspective d'un mot désignant les habitants des gebels/déserts. Tout au long des époques tardives on peut glaner des attestations de cette appellation réduites au signe du terrain vallonné suivi par les

\footnotetext{
${ }^{15}$ Voir Chr. Desroches-Noblecourt - Ch. Kuentz, Le Petit Temple d'Abou Simbel, I, Le Caire 1968, p. 149, n. 70 ; C. PEUST, loc. cit.

${ }^{16}$ Voir $W b$. III 234, 14 ; P. WiLSON, A Ptolemaic Lexikon (OLA 78), Louvain 1997, p. 705.

${ }^{17}$ Voir Fr. Colin, op. cit., pp. 342-343, n. III. Proposition reprise par I. GuermeUR, Les Cultes d'Amon hors de Thèbes (BEPHE 123), Turnhout 2005, p. 425, J. MoJE, op. cit., pp. 27, 211 et 421 422 (pour les exemples saïtes) et Fr. PAYRAUDEAU, L'Egypte et la vallée du Nil, III : Les époques tardives (1069-332 av. J.-C.), Paris 2020, p. 269.

${ }^{18}$ Cf. infra, pp. 88-90.

${ }^{19} C f$. Fr. Colin, dans D. Devauchelle (éd.), La XXVI dynastie, continuités et ruptures : Actes du Colloque international organisé les 26 et 27 novembre 2004 à l'Université Charles-de-GaulleLille 3, Paris 2011, p. 47, n. 3.
} 
trois traits du pluriel. On en trouve dès l'époque libyenne ${ }^{20}$ même si la plupart des exemples se concentrent au $\mathrm{IV}^{\mathrm{e}}$ siècle avant notre ère ${ }^{21}-$ donc à l'époque d'Ounamon - et un peu plus tard ${ }^{22}$. En apparaissant comme la seule susceptible de convenir à l'ensemble des exemples, cette troisième possibilité invite même à exclure les précédentes.

Ainsi comprise, la désignation des maîtres de Siwa peut être rapprochée d'un titre attesté à l'époque libyenne qui se présente sous la forme $w r{ }^{3}$ h hstyw, son

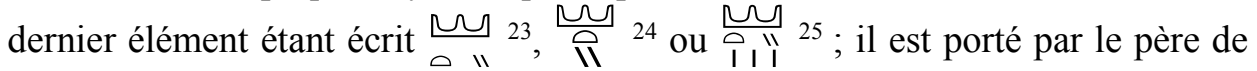
Patarechnes, la mère de Nimlot $\mathrm{B}$, que nous connaissons grâce aux monuments de ce dernier. On le retrouve aussi dans l'allusion aux $w r(w) n h 3 s t y w$ de la grande inscription d'Osorkon $\mathrm{I}^{\mathrm{er}}$ à Bubastis où h3styw est écrit $\left.\frac{\Omega}{011}\right|_{\frac{1}{1}}{ }^{26}$, qui devient II dans la version parallèle livrée par une statue d'Osorkon II $^{27}$. Sans doute est-il encore présent à la fin de la Troisième Période intermédiaire sur la fameuse effigie de Bepeches où il se réduit à ${ }^{28}$. Malgré une ressemblance formelle, ce titre ne doit pas être confondu avec celui attesté à Siwa dans la mesure où il est employé en Égypte pour désigner dans l'armée les chefs des mercenaires levés à l'extérieur du pays ${ }^{29}$. Sinon la désignation des maîtres de Siwa rappelle aussi bien par son aspect que par son sens les mentions des wrw h3styw relevées dans les témoignages relatifs aux campagnes militaires du Nouvel Empire, où cette expression s'applique aux chefs des étrangers que combat le pharaon ${ }^{30}$.

D'un point de vue plus général on peut noter que le terme h3styw entre dans la composition de bien d'autres titres à la Basse Époque, la plupart concernant des

\footnotetext{
${ }^{20}$ Voir K. JANSEN-Winkeln, Ägyptische Biographien der 22. und 23. Dynastie (ÄAT 8), Wiesbaden 1985 , p. 532 , c. 10.

${ }^{21}$ Voir G. Lefebvre, Le Tombeau de Pétosiris, II, Le Caire 1923, p. 32, 59.3 ; D. KLotz, BIFAO 110 (2010), p. 147.

${ }^{22}$ Voir Urk. II 38, 8 ; Edfou VI 300, 5.

${ }^{23}$ Voir E. Rogge, Statuen des Neuen Reiches und der Dritten Zwischenzeit (CAA Kunsthistoriches Museum Wien 6), Mayence 1990, pp. 158, 31, et 157, 26 (2 ex.).

${ }^{24}$ Voir K. JANSEN-WINKELN, Inschriften der Spätzeit, II, Wiesbaden 2007, p. 85, 17.3 (2 ex.).

${ }^{25}$ Voir ID., Orientalia 75 (2006), p. 314, col. 6 (1 ex.).

${ }^{26}$ Voir E. LANGE, ZÄS 135 (2008), pl. XXXVII, 1. x+7.

27 Voir H. JACQUET-Gordon, JEA 46 (1960), p. 16, 1. 9 du texte.

${ }^{28}$ Voir O. Perdu, Les Statues privées de la fin de l'Égypte pharaonique (musée du Louvre), I, Paris 2012, p. 434.

${ }^{29}$ Suivant l'interprétation de J. YoyotTe, dans Mélanges Maspero, $\mathrm{I}^{4}$ (MIFAO 61), Le Caire 1961, p. 142 et n. 4.

${ }^{30}$ Voir e. g. KRI II 180, 8 ; V 56, 8.
} 
fonctions exercées par des militaires dans le cadre de l'armée. Celles-ci sont définies par les mots ou expressions $m r$, « directeur $»^{31}, m r-m \varsigma^{\longleftarrow}$, « général $»^{32}, h q$ 3, « régent $»^{33}$, hrp, «administrateur $»^{34}$, ou $s \check{m} m$, « conducteur $»^{35}$. Les graphies habituellement employées dans ces titres pour désigner les «gens des gebels/

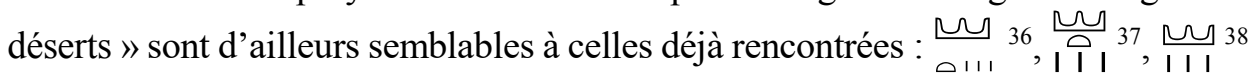
et $\stackrel{\simeq}{\simeq}{ }^{39}$; on en compte même une où le sens de ḩ3styw est clairement exprimé en

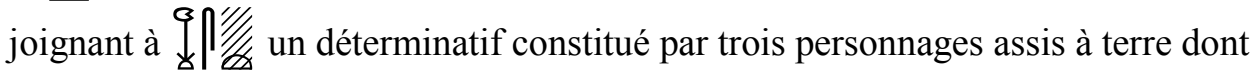
les genoux sont surmontés par une plume marquant leur caractère libyen ${ }^{40}$. Dans ce contexte la désignation des «gens des gebels/déserts» s'applique plus précisément aux mercenaires enrôlés dans l'armée égyptienne ${ }^{41}$, comme c'est aussi le cas dans les références aux «chefs » des ḩstyw relevées précédemment dans le Delta. En ce qui concerne l'appellation du maître de Siwa en revanche, le mot conserve son sens initial en s'appliquant aux populations locales qu'il décrit comme celles qui habitent des contrées vallonnées et arides, contrairement aux Égyptiens qui occupent la partie plate et fertile le long de la basse vallée du Nil.

${ }^{31}$ Voir A. Rowe, $A S A E ́$ 38 (1938), p. 187 et pl. XXIV. Le titre peut être complété par une précision sur la nature des contingents étrangers concernés ; voir G. BOTTI - P. ROMANELLI, Le sculture del Museo Gregoriano Egizio, Vatican 1951, pl. VI (H3w-nbw); E. BRESCIANI, SCO 16 (1967), pl. V, col. 1 du texte (Sttyw); L. Gestermann, RdÉ 52 (2001), pl. XXII, 1. 3 du texte (Thhnw) ; K. JANSEN-WinKELN, Inschriften der Spätzeit, IV, Wiesbaden 2014, p. 784, 60.78 (hr-ib W3 $\underline{d}-w r)$.

32 Voir P. E. Newberry, Funerary Statuettes and Model Sarcophagi (CGC), I, Le Caire 1937, pp. 138-139, n 47363-47365 ; III, Le Caire 1957, pl. XLII, n 47363.

${ }^{33}$ Voir Urk. II 24, 7 (où le titre est suivi par $m$ Hnt-ỉ btt), en joignant les exemples ne concernant pas des militaires mais deux majordomes de l'adoratrice thébaine dans E. GRAEFE, Untersuchungen zur Verwaltung und Geschichte der Institution der Gottesgemahlin des Amun vom Beginn des Neuen Reiches bis zur Spätzeit (̈̈gAbh 37), I, Wiesbaden 1981, pp. 21-22, j65, et 77, p211 (où le titre est suivi par $m W 3 s t$ ).

${ }^{34}$ Voir Fr. W. von Bissing, Steingefässe (CGC), Vienne 1904, p. 157. Le titre peut être complété par une précision sur la nature des contingents étrangers concernés ; voir K. JANSEN-WINKELN, op . cit., pp. 491, 57.190, 492, 57.195, et 493, 57.199 (rsyw) ; C. PRICE, dans Cl. JURMAN - B. BADER D. A. Aston (éds), A True Scribe of Abydos: Essays on First Millenium Egypt in Honour of Anthony Leahy (OLA 265), Louvain-Paris-Bristol 2017, p. 383 (H3w-nbw).

${ }^{35}$ Voir H. De Meulenaere, BIFAO 63 (1965), pp. 21, I.B, et 23, III.5.

${ }^{36}$ Voir A. Rowe, loc. cit. ; H. De Meulenaere, op. cit., p. 21, I.B.

37 Voir Fr. W. vON BISSING, loc. cit.

${ }^{38}$ Voir P. E. Newberry, loc. cit. ; H. De Meulenaere, op. cit., p. 23, III.5 ; C. Price, loc. cit.

${ }^{39}$ Voir Urk. II 24, 7 ; G. Botti - P. Romanelli, loc. cit. ; E. Bresciani, loc. cit. ; E. Graefe, loc. cit.; K. JANSEN-WINKELN, loc. cit.

${ }^{40}$ Voir L. GESTERTMANN, loc. cit.

${ }^{41} C f$. S. Sauneron - J. Yoyotte, BIFAO 50 (1952), p. 171, n. 2 ; D. A. Pressl, Beamte und Soldaten: Die Verwaltung in der 26. Dynastie in Agypten (664-525 v. Chr.), Francfort-sur-le-Main 1998, pp. 90-91, § 8.1.2. 
Sur le plan politique le recours au mot $w r$ pour désigner le maître de Siwa revêt un intérêt particulier. Parmi ses divers emplois, l'un consiste précisément à l'appliquer à tout souverain d'une contrée ou d'une localité en dehors du territoire égyptien, suivant une acception qui en fait l'équivalent de Šarru, « roi », dans les textes en babylonien ${ }^{42}$. Cet usage abondamment illustré au Nouvel Empire ${ }^{43}$, notamment par les témoignages relatifs aux campagnes militaires ou les archives diplomatiques d'Amarna ${ }^{44}$, perdure aux époques tardives comme le reflète en particulier la façon de qualifier le souverain achéménide de $p 3$ wr $n n 3$ wrw, « roi des rois $»^{45}$.

Ce qu'il en est de la situation de Siwa à la $\mathrm{XXVI}^{\mathrm{e}}$ dynastie est particulièrement révélateur si nous la comparons à celle de Bahariya, dont on est assez bien informé à cette période. Dans cette oasis autrement plus proche de la vallée du Nil, le pouvoir est entre les mains d'une lignée de personnages dont l'autorité s'exprime à travers le titre « comte-gouverneur de l'oasis » $\left(r p^{\ulcorner} h 3 t y-^{\top}{ }^{\top} w h 3 t\right)^{46}$, le plus connu étant un contemporain de la fin de l'époque saïte nommé Djedkhonsouiouefânkh ${ }^{47}$. Il s'agit donc d'agents du pouvoir pharaonique intervenant dans une région soumise à son autorité, tout comme leurs homologues du Nouvel Empire ${ }^{48}$, quand la situation rencontrée à Bahariya n'était pas différente de celle observée dans les établissements nubiens sous domination égyptienne ${ }^{49}$. La sujétion des gouverneurs de Bahariya au pharaon saïte se reflète dans le décor des chapelles édifiées à 'Ayn el-Mouftella à la fin de la XXVI ${ }^{\mathrm{e}}$ dynastie ${ }^{50}$. Si Djedkhonsouiouefânkh est parfois représenté, il ne l'est jamais autant qu'Amasis, derrière lequel il vient

${ }^{42}$ Cf. W. L. MoRAn, The Amarna Letters, Baltimore-Londres 1992, p. XXVII, avec n. 73. Le mot $w r$ peut d'ailleurs être employé en parallèle avec $n s w$ comme c'est le cas dans le conte opposant Apophis et Séqenenrê ; $c f$. A. PAULET, BSÉG 27 (2005-7), pp. 76-77.

${ }^{43}$ Antérieurement le terme habituellement utilisé à ce propos est $h q 3 ; c f$. G. POSENER, Princes et pays d'Asie et de Nubie, Bruxelles 1940, p. 41. Dès le Moyen Empire il y a néanmoins quelques exemples de $w r$ de tribu nubienne ; $c f$. IBID., pp. 41 et 88-89, E 50 et E 51.

${ }^{44} C f$. D. LORTON, The Juridical Terminology of International Relations in Egyptian Texts through Dyn. XVIII, Baltimore-Londres 1974, pp. 60-63, § A ; A. SPALINGER, SAK 9 (1981), p. 302, n. 10.

${ }^{45}$ Voir G. Posener, La Première Domination perse en Égypte: Recueil d'inscriptions hiéroglyphiques (BdÉ 11), Le Caire 1936, pl. IV, 1. 4 du texte, et V, 1. 4 du texte ; J. YoYOTTE, Journal Asiatique 260 (1972), p. 255, 2.4.

${ }^{46} C f$. Fr. Colin - Fr. LABriQue, dans Fr. LABrique (éd.), Religions méditerranéennes et orientales de l'Antiquité : Actes du colloque des 23-24 avril 1999, Institut des sciences et techniques de l'Antiquité (UMR 6048), Université de Franche-Comté, à Besançon (BdÉ 135), Le Caire 2002, p. 71.

${ }^{47}$ Titulatures mentionnées IBID., pp. 60, 65 et 67.

${ }^{48}$ Cf. L. L. GidDY, Egyptian Oases : Bahariya, Farafra, Dakhla and Kharga During Pharaonic Times, Warminster 1987, pp. 162-163.

${ }^{49} C f$. Br. Gratien, dans C. Berger et al. (éd.), Hommages à Jean Leclant, II (BdÉ 106/2), Le Caire 1994, p. 188.

${ }^{50}$ Voir A. FAKHrY, Bahria Oasis, I, Le Caire 1942, pp. 151-171 et pl. XLIII-LXII. 
d'ailleurs se placer la seule fois où ils sont figurés côte à côte ${ }^{51}$. En ce qui concerne le maître de Siwa à la même période, il bénéficie d'un statut bien différent puisqu'il peut se prévaloir d'un titre employé par les Égyptiens pour désigner les souverains étrangers. Son importance ressort de la façon dont Sethirdis est présent dans le saint des saints d'Aghourmi, la seule partie décorée du temple. De chaque côté de l'entrée apparaît un personnage qui est confronté à quatre paires de divinités se succédant le long des murs latéraux, la série orientale (côté vallée du Nil) débutant avec l'Amon thébain (à tête humaine) et l'autre (côté désert libyque) avec l'Amon local (à tête de bélier) ${ }^{52}$. Or, suivant une répartition conforme à la logique géographique, les personnages qui font face à ces deux formes d'Amon sont respectivement Amasis et Sethirdis ${ }^{53}$, lequel se trouve ainsi traité sur un pied d'égalité avec le pharaon. C'est d'ailleurs d'autant plus frappant que les restes de deux légendes, la sienne ${ }^{54}$ et celle de la troisième divinité du mur est ${ }^{55}$, $\mathrm{s}^{\prime}$ accordent pour le qualifier de « roi de Haute et Basse Égypte » avant de mentionner - comme dans les autres passages où il est cité ${ }^{56}$ - son titre, son nom et éventuellement son père, comme s'il s'agissait d'un simple particulier. Le personnage n'en jouit pas moins d'un traitement privilégié dans la mesure où il est le seul bénéficiaire des bienfaits qu'au moins six des divinités représentées sont censées accorder ${ }^{57}$.

La façon dont Ounamon nous apparaît à la $\mathrm{XXX}^{\mathrm{e}}$ dynastie montre une plus grande volonté du maître de Siwa de s'assimiler à un pharaon. Cette tendance est particulièrement nette dans le petit temple d'El-Bahreïn, à un peu plus d'une centaine de kilomètres à l'est de Siwa. Suivant un aménagement rappelant le saint des saints d'Aghourmi, aux scènes de la partie orientale où Nectanébo I ${ }^{\mathrm{er}}$ joue le rôle de l'officiant, notamment devant l'Amon thébain, correspondent à l'ouest d'autres scènes où ce rôle est cette fois tenu par Ounamon, qui intervient en

\footnotetext{
${ }^{51}$ Voir IBID., pp. 161-162 et pl. LIV.A.

52 Voir A. FAKHRY, Siwa Oasis, pp. 91-95, en joignant les commentaires de Fr. Colin, dans Studies Dedicated to the Memory of Jan Quaegebeur, I, pp. 336-339, et I. Guermeur, op. cit., pp. 424-425.

${ }^{53}$ Voir A. FAKHRY, op. cit., p. 91. De sa représentation il ne reste que la plume libyenne plantée au sommet de son crâne.

${ }^{54}$ Voir $I B I D$.

55 Voir Kl. P. KuHLMAnN, MDAIK 57 (2001), p. 199.

${ }^{56}$ Voir A. FAKHRY, op. cit., pp. 92-95, avec cinq exemples dont trois comportent l'indication du père.

${ }^{57}$ Voir A. FAKhrY, op. cit., pp. 92 (Amon thébain), 93 (Mahès ou Amon? et Amon de Siwa), 94 (Paherenimen) et 95 (Hérichef) ; K1. P. KuHLMANN, loc. cit. (Chnoum). Les légendes de ces divinités mentionnent en effet leur nom et ce qu'elles donnent à Sethirdis en étant conçues sur le modèle des inscriptions gravées sur les ex-voto à l'effigie de divinités ; $c f$. J.-M. KRUCHTEN, JEA 82 (1996), pp. 60-63.
} 
particulier devant l'Amon local ${ }^{58}$. Or cette personnalité de Siwa adopte une apparence dont quelques détails le rapprochent du pharaon. Non seulement il porte une barbe postiche et un pagne à devanteau comme Nectanébo I ${ }^{\mathrm{er}}$, mais il a la tête ceinte d'un bandeau à l'avant duquel est fixée la plume libyenne, laquelle se dresse au-dessus de son front comme un urceus $^{59}$. Par ailleurs il se compose une titulature dont le contenu et la forme imitent celles des pharaons ${ }^{60}$. D'un côté il fait précéder son titre de wr h3styw et son nom de deux épithètes inspirées par les protocoles royaux pharaoniques: $\mathrm{Hr} \underline{t} \mathrm{t}_{3} \mathbf{-}^{-}$, « l'Horus au bras vaillant», nom d'Horus de Nectanébo $\mathrm{I}^{\text {er }}$ se référant à l'Horus libyen ${ }^{61}+w^{\complement} f$ h h3sw.t imntyw $(t)$ (ou ḩstyw imntyw) ${ }^{62}$, «celui qui soumet les déserts/pays occidentaux (ou les gens des déserts/pays occidentaux) $»^{63}$, épithète à rapprocher de $w^{\complement} f$ h hiswt dans les titulatures de Séthi $\mathrm{I}^{\text {er }}{ }^{64}$, Ramsès $\mathrm{II}^{65}$ et Téôs ${ }^{66}$ qui définit l'un des rôles

${ }^{58} C f$. P. GALlo, op. cit., p. 19. D’un point de vue plus général on peut observer que Nectanébo ${ }^{\text {er }}$ et Ounamon officient respectivement devant des divinités qui sont majoritairement de la vallée du Nil ou de la région de Siwa; en ce qui concerne le pharaon, voir L. UGGETTI, dans P. GALLO (éd.), Egittologia a Palazzo Nuovo : Studi e ricerche dell'Università di Torino, Novi Ligure 2013, pp. 280, fig. 15 (Amon thébain), 281, fig. 16 (Horus de Létopolis), 282, fig. 20 (Ptah de Memphis); quant au maître de Siwa, voir IBID., pp. 277, fig. 8 (Amon d'El-Bahreïn), 283, fig. 23 (Osiris d'El-Bahreïn), 284, fig. 26 (Horsaiset présent à Siwa), et 286, fig. 31 (Paherimen et Tefnout de Siwa).

${ }^{59}$ Voir P. GALlo, op. cit., pp. 24, fig. 11, 25, fig. 12, et 26, fig. 13-14.

${ }^{60}$ Voir IBID., pp. 25, fig. 12, et 26, fig. 14 ; L. UGGETTI, op. cit., pp. 281, fig. 17, et 283, fig. 24.

${ }^{61} C f$. P. Gallo, op. cit., p. 25, n. 18 ; Kh. El-EnANy, BSÉG 30 (2014-15), pp. 6-7.

${ }^{62}$ En admettant que $\int$ vaut pour ${ }_{f}$, avec l'emploi de la partie significative du signe pour l'ensemble (pars pro toto), suivant un procédé attesté notamment avec 2 pour 7 , dont les exemples se limitent aux époques les plus récentes, les premiers remontant précisément aux dernières dynasties indigènes; $c f$. D. MEEKS, Les Architraves du temple d'Esna: Paléographie (PalHiéro 1), Le Caire 2004, p. 95, § 256 ; Å. EngShEden, Le Naos de Sopdou à Saft el-Henneh (CG 70021) : Paléographie (PalHiéro 6), Le Caire 2014, p. 59, § 155.

${ }^{63}$ Cette proposition diffère de celle de P. Gallo, op. cit., p. 25, qui suggère de lire wef nht h3swt $\breve{S} w$ et de comprendre « celui qui soumet, le puissant des déserts de Chou » (rapproché de nht ḩ3swty $n \breve{S} w$ dans un passage très lacuneux dont la lecture demeure problématique), en ne reconnaissant pas dans le signe du bras tenant un bâton le déterminatif habituel de w'f. Suivant cette nouvelle interprétation l'épithète pourrait se faire l'écho de la nécessité de s'imposer dans une région peu sûre, où les sources classiques évoquent un quotidien marqué par les pillages ; $c f$. Fr. CoLIN, Les Peuples libyens de la Cyrénä̈que à l'Égypte d'après les sources de l'Antiquité classique, Bruxelles 2000, pp. 108-115, développant un point de vue déjà exprimé par Fr. CHAmoux, Cyrène sous la monarchie des Battiades, Paris 1953, p. 59, à propos de la période postsaïte.

${ }^{64}$ Voir J. vON BECKERATH, Handbuch der ägyptischen Königsnamen (MÄS 49), Mayence 1999, p. $151, \mathrm{~N} 5$.

${ }^{65}$ Voir IBID., p. 155, G 9

${ }^{66}$ Voir A. I. BlÖBAum, ,Denn ich bin ein König, der die Maat liebt“ Herrscherlegitimation im spätzeitlichen Ägypten: Eine vergleichende Untersuchung der Phraseologie in den offiziellen Königsinschriften vom Beginn der 25. Dynastie bis zum Ende der makedonischen Herrschaft (Aegyptiaca Monasteriensia 4), Aix-la-Chapelle 2006, p. 409. 
traditionnellement dévolus au pharaon parallèlement à la protection du pays ${ }^{67}$. De l'autre il répartit cet ensemble dans des cartouches, le dernier étant destiné à son titre et à son nom; il y en a généralement deux, respectivement introduits par les titres « roi de Haute et Basse Égypte » et « fils de Rê ${ }^{68}$, mais on peut en prévoir trois $^{69}$ en réservant les deux premiers aux épithètes pour éviter leur entassement dans un seul ${ }^{70}$ (fig. 3). Ce souci d'Ounamon de se présenter tel un souverain égyptien se reflète encore, mais à un degré moindre, dans les quelques images que les maigres vestiges du temple d'Oum-Ebeyda conservent de lui à côté d'une seule du pharaon contemporain. Les signes de cette volonté se limitent cette fois au port du pagne à devanteau ${ }^{71}$, à la plume frontale pastichant l'urceus royal ${ }^{72}$ et à l'insertion de l'épithète le définissant comme «l'Horus au bras vaillant » en tête de sa titulature qui, pour le reste, ne se différencie pas de celles des particuliers en mentionnant son titre et son nom suivis de l'indication de ses parents ${ }^{73}$.

Fig. 3 : Version de la titulature d'Ounamon avec trois cartouches dans le temple d'El-Bahreïn (d'après P. GALLO, BSFÉ 166 [juin 2006], p. 26, fig. 14)

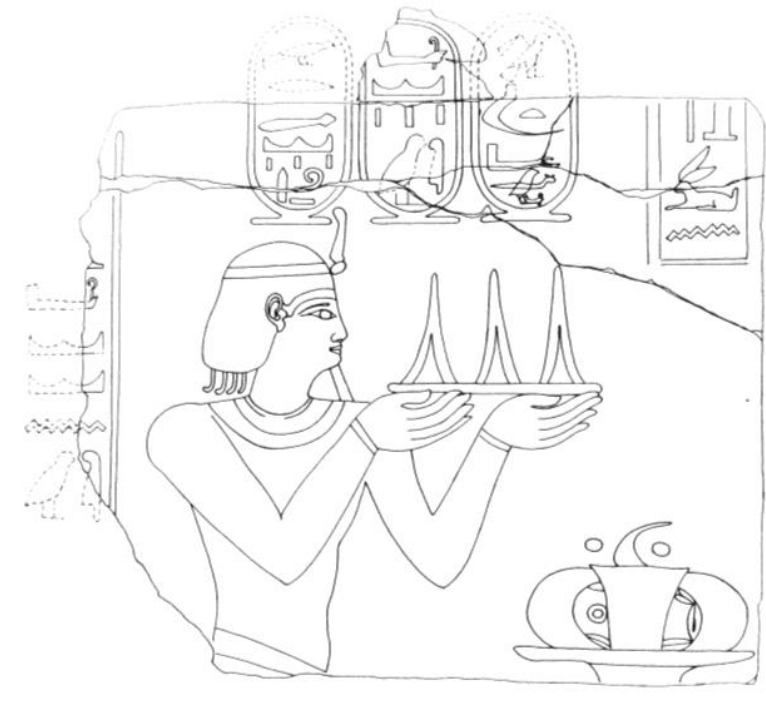

${ }^{67} \mathrm{Cf}$. N.-Chr. GRIMAL, Les Termes de la propagande royale égyptienne de la XIX dynastie à la conquête d'Alexandre, Paris 1986, pp. 678-679.

${ }^{68}$ Voir P. Gallo, op. cit., p. 25, fig. 12 ; L. UGGetTI, loc. cit.

${ }^{69}$ Voir P. Gallo, op. cit., p. 26, fig. 14.

${ }^{70}$ Le problème posé par l'insertion des épithètes dans un seul cartouche est illustré par l'exemple reproduit $I B I D$., p. 25 , fig. 12 , où cela a conduit à réduire la seconde à $w^{\complement} f$ suivi directement par imntyw, ce dernier mot étant noté uniquement par une plume, laquelle est reportée faute de place devant le titre « fils de Rê ».

${ }^{71}$ Voir A. FAKHRY, op. cit., p. 117, fig. 2.

72 Voir P. Gallo, op. cit., p. 28, fig. 15 ; K1. P. KuHLMAnN, ASAÉ 88 (2008), p. 196, fig. 7 ; ID., $A S A E ́$ 84 (2010), pp. 229, fig. 13, et 241, fig. 7.

${ }^{73}$ Voir P. GALLO, op. cit., p. 28, fig. 16. 
Dans une région où l'influence pharaonique s'observe aussi bien dans l'onomastique que dans les cultes ou l'aménagement des édifices religieux, ce que nous entrevoyons des maîtres de Siwa par le biais de leurs signalements révèle une situation assez singulière qui a perduré par-delà la période où l'Égypte et la Libye ont été réunies dans une satrapie - la sixième - dominée par le Grand Roi perse. D'une part ils se prévalent d'un titre les désignant comme de véritables rois au niveau local, allant même jusqu'à se donner des allures de pharaon en empruntant à leur modèle certaines de ses prérogatives. D'autre part ils n'en restent pas moins des personnages respectueux de l'autorité pharaonique, dont on indique le titre, le nom et les parents comme on le ferait pour un simple particulier. À travers la façon dont ils se présentent, Siwa et sa région peuvent donc apparaître comme un territoire administrativement souverain malgré ses liens étroits avec l'Égypte, ce qui peut nous rappeler, toute proportion gardée, la position de la principauté de Monaco vis-à-vis de la France telle qu'elle ressort du traité conclu entre les deux pays le 17 juillet 1918. Les liens de Siwa avec l'Égypte se reflètent assez bien dans la façon dont les temples d'Aghourmi et El-Bahreïn mettent en scène le pharaon et l'autorité locale. En les voyant officier parallèlement, répartis équitablement sur les deux côtés du sanctuaire, on mesure en effet leur parfaite complémentarité. Le fait que le pharaon soit représenté à Siwa alors que son représentant ne l'est pas en Égypte révèle toutefois un déséquilibre dans leur relation qui se fait au profit du plus fort. On perçoit ainsi dans leur rapprochement un moyen pour l'Égypte de renforcer son emprise à l'Ouest sans recourir à la force et pour Siwa une façon de composer avec un puissant voisin, quitte à admettre son ascendant, dans la mesure où il ne cherche pas à menacer son intégrité en étant même susceptible de constituer une protection. En se limitant surtout à la Cyrénaïque, notre connaissance des événements ayant marqué l'évolution du désert libyque durant la Basse Époque ne permet malheureusement pas d'appréhender la situation avec plus de précision. On peut seulement constater que l'immigration grecque encouragée à Cyrène dès l'époque d'Apriès - avec l'hostilité qu'elle a suscitée de la part des populations locales - était de nature à inciter les Libyens à se rapprocher des Égyptiens ${ }^{74}$.

\footnotetext{
${ }^{74}$ Cf. Fr. Chamoux, op. cit., p. 135.
} 